\title{
Transition waves and nonlinear interactions in the near wake of a circular cylinder*
}

\author{
LING Guocan (凌国灿), Allen T. Chwang ${ }^{* *}$, NIU Jiayu (牛家玉) ${ }^{* *}$ \\ and WANG Dongjiao (王冬婈) ** \\ (Laboratory for Nonlinear Mechanics of Continuous Media, Institute of Mechanics, \\ Chinese Academy of Sciences, Beijing 100080, China)
}

Received January 25, 1997

\begin{abstract}
Transition waves and interactions between two kinds of instability — vortex shedding and transition wave in the near wake of a circular cylinder in the Reynolds number range 3000-10 000 are studied by a domain decomposition hybrid numerical method. Based on high resolution power spectral analyses for velocity new results on the Reynolds-number dependence of the transition wave frequency, i. e. $f_{t} / f_{s} \sim R e^{0.87}$ are obtained. The new predictions are in good agreement with the experimental results of Wei and Smith but different from Braza's prediction and some early experimental results $f_{\mathrm{t}} / f_{\mathrm{s}} \sim R e^{0.5}$ given by Bloor et al. The multi-interactions between two kinds of vortex are clearly visualized numerically. The strong nonlinear interactions between the two independent frequencies $\left(f_{\mathrm{t}}, f_{\mathrm{s}}\right)$ leading to spectra broadening to form the coupling $m f_{\mathrm{s}} \pm n f_{\mathrm{t}}$ are predicted and analyzed numerically, and the characteristics of the transition are described. Longitudinal variations of the transition wave and its coupling are reported. Detailed mechanism of the flow transition in the near wake before occurrence of the threedimensional evolution is provided.
\end{abstract}

Keywords: transition wave, nonlinear interaction, near wake, circular cylinder.

The transition phenomenon in the near wake of a circular cylinder is the mechanisms of the onset of turbulence, which have been studied by many experimentalists, such as Crausse ${ }^{[1]}$, Roshko $^{[2]}$, Gerrard ${ }^{[3]}$, Bloor ${ }^{[4]}$ and recently by Wei and Smith ${ }^{[5]}$, Kourta et al . ${ }^{[6]}$. Bloor pointed out that as Reynolds number increases, especially for that higher than 2000 this phenomenon becomes prominent, and first provided a relation between the ratio of the transition wave frequency over the vortex shedding frequency and Reynolds number, i. e. $f_{\mathrm{t}} / f_{\mathrm{s}} \sim R e^{0.5}$. This influence of Reynolds number on the position of the transition was also discussed. This relation was also considered by Kourta et al. ${ }^{[6]}$. The transition phenomena were also confirmed by a more recent study of Wei and Smith in the Reynolds number range $1200-11000$. But the dimensionless transition wave frequency demonstrated a 0.87 power-law relationship relative to Reynolds number, contrary to the 0.5 power-law given by Bloor ${ }^{1}$. All the previous experimental results suggest that the transition process in the near wake was mainly governed by the interactions between the large-scale alternate vortices in the wake and small eddies in the mixing layer and characterized by spectral broadening. The two kinds of instability- the vortex shedding and the transition wave

* Project supported by the National Natural Science Foundation of China, the LNM of Institute of Mechanics, and partially by the National Basic Research Project.

* * Department of Mechanical Engineering, University of Hong Kong, Hong Kong.

** Institute of Mechanics, Chinese Academy of Sciences, Beijing 100080, China.

1) As the authors finished checking-up the final proof of the present paper they found a new result published by Prasad \& Williamson (Phys. Fluids, June 8,1996 ), who found that $f_{\mathrm{t}} / f_{\mathrm{s}} \sim R e^{0.67}$ by measurements and data analysis. 
initially are two-dimensional. However, the basic features concerning the Reynolds number dependence of the nondimensional transition wave frequency obtained by the previous studies do not coincide with each other. Different rationales and arguments were put forward in separate studies. They are still not adeguately understood. Moreover there is only little information on the spatial variation of the transition wave and nonlinear interaction between the two kinds of instability as Reynolds number varies.

On the other hand, very few numerical studies have been devoted to the transition features. The first prediction of the transition phenomena behind the cylinder was given by Braza $e t a l^{[7]}$. by solving the two-dimensional time-dependent Navier-Stokes (N-S) equations in the Reynolds number range $2000-10000$. Their results confirmed that before the occurrence of the three-dimensional evolution of the near wake the two-dimensional instability in the shear layer develops and leads to generation of small eddies. The calculated ratio of the transition wave frequency was close to the experimental result of Bloor. The interactions of two kinds of vortex and the generation of sideband $m f_{\mathrm{s}} \pm n f_{\mathrm{t}}$ were also revealed. In their computations the mesh system used was probably not fine enough and the frequency resolution used was low, only 0.019 5. These will lead to some limitations in accurate evaluation of peak values corresponding to different structures. Besides, the spatial variation of the transition wave and the strong nonlinear interaction characterizing flow transition has not been reported. Therefore it is necessary to make further study on the basic features of the transition waves and the spatial variation of the transition properties. It may also be helpful for clearing up some discrepancies in the previous experimental results as mentioned above. In the present work the transition characteristics of near wake behind the cylinder in the Reynolds number range $3000-10000$ will be investigated in detail by numerical visualization of flow evolution and spectral analyses for velocities.

\section{Numerical method}

\subsection{Numerical model}

For the above purpose accurate numerical solutions with high resolution for the near wake flow are required. Also a large amount of numerical signals of velocity or pressure over a sufficiently long period of vortex shedding at various positions along the separated shear layer should be used for obtaining a higher resolution in the spectral analysis. Thus the finite difference numerical solutions for the steady N-S eqnations will consume a large amount of CPU time and memories, especially for large Reynolds numbers. Consider the fact that the flow evolution and the transition phenomena to be studied in detail occur mainly in the formation region of the wake vortex. The conventional length scale of the region is roughly estimated to be only several radii long behind the cylinder by experimental visualization ${ }^{[6]}$. Moreover, as Reynolds number increases the length of the dead-fluid zone decreases and the location of occurrence of the instability in mixing layer moves upstream. Therefore to overcome the difficulties of large memory and CPU time in solving the $N$ $\mathrm{S}$ equations, we will confine the domain of integration of the N-S equations in the small region near the cylinder surface, and use very dense mesh system and proper boundary conditions to solve the N-S equations. In the meantime we calculate the vorticity convection in the other region by a proper vortex method with considerable accuracy, and evaluate the global features of the vortex shedding flow. The flows in the two regions are solved simultaneously. 
Based on the above idea, by means of a domain decomposition hybrid method suggested by Ling et al.$^{[8,9]}$, the entire flow field is divided into an inner domain, of the order of the cylinder's radius, near the cylinder surface, in which the flow is viscous, and an outer one at rest, where the flow is assumed approximately to be inviscid. There is a transition region, in brief, an interface between the two domains. The size of the inner domain can be adjusted depending on how detailed information is required for various flow parameters and CPU time limit. The flow in the inner domain will be determined by an unsteady vorticity transport equation and a Poisson equation of stream function. These two equations are solved by the finite difference method using a very fine mesh system. The vorticity in the outer region flow is obtained from vorticity flux across the interface and is converted into nascent discrete vortex. At higher Reynolds numbers the convection of the vortices is predicted by the Lagrange-Euler vortex-in-cell method (VIC) proposed by Stansby \& Dixon ${ }^{[10]}$ and calculated in a coarse mesh system available in the entire flow field. The convection velocity of the vortex particles is determined through solving the Poisson equation of stream function by vorticity distributed in the entire flow field and boundary conditions at the cylinder surface as well as at infinity.

The boundary condition, i.e. the vorticity values at the interface, $\omega_{I}$, for solving the vorticity transport equation in the inner domain may be given by careful interpolation of vorticity on both sides of the interface. That is,

$$
\omega_{I}(i, j)=\sum_{k=I-N}^{I+N} \Delta \omega_{k}(i, j)
$$

where $\omega_{I}(i, j)$ is the vorticity value at mesh point $(i, j)$ on the interface $I . \Delta \omega_{k}(i, j)$ is the interpolation components onto the interface of vorticity of discrete vortices. These vortices are initially, say, at the previous time step, located at the $k$ th mesh layer near the interface and move for a time step. $N$ is a positive integer greater than 1 . The stream function values at the interface $\psi_{I}$ are also given by interpolation of nearby stream functions. Across the interface conservation of flow flux holds. Thus in a time interval we first find out a fine solution for the inner flow, then calculate the vortex movement in the outer region in a coarse mesh-point system. Again we can obtain the boundary conditions for the inner solution at the next time step, and then go on with the calculation.

\subsection{Numerical schemes for the inner and outer domain flows}

By a transformation of $r=\exp (2 \pi \xi), \theta=2 \pi \eta$ the flow in physical plane $(r, \theta)$ is transformed into a calculation plane $(\xi, \eta)$. The governing equations for the inner region flow are vorticity equation and Poisson equation for the stream function. The non-dimensional divergence forms may be represented as

$$
E \frac{\partial \omega}{\partial t}+\frac{\partial}{\partial \xi}(U \omega)+\frac{\partial}{\partial \eta}(V \omega)=\frac{2}{R e}\left(\frac{\partial^{2} \omega}{\partial \xi^{2}}+\frac{\partial^{2} \omega}{\partial \eta^{2}}\right), \Delta \psi=-E \omega,
$$

where $E=4 \pi^{2} \exp (4 \pi \xi), U=E^{\frac{1}{2}} v_{r}, V=E^{\frac{1}{2}} v_{\theta}, \quad v_{r}$ and $v_{\theta}$ are dimensionless velocity components in physical plane $(r, \theta)$ normalized by $U_{\infty} . t=\frac{t^{\prime} U_{\infty}}{R}$ and $t^{\prime}$ is physical time. Here Reynolds number is defined by $2 U_{\infty} R / \nu$, and $\nu$ is the dynamic viscosity coefficient. At the cylinder surface the no-slip condition is satisfied. The boundary conditions at the interface are giv- 
en by formula (1). The initial conditions are $t=0, \omega=0$ and $\psi=0$, i. e. it is a potential solution.

The convection part of the vorticity transport equation in (2) is solved by a second-order accurate scheme of central difference and the alternating direction $(\xi, \eta)$ implicit algorithm (A.D. I. ). Each time-step $\Delta t$ is decomposed into two successive half-steps with second-order accuracy.

The total order of accuracy of this scheme is $O\left((\Delta \xi)^{2},(\Delta \eta)^{2}, \Delta t\right)$. The Poisson equation in (2) is solved by the fast Fourier transformation algorithm (FFT). All the above computations are performed in a fine mesh-point system.

Vortex movement in the outer domain is calculated by the Lagrange-Euler vortex method. The strength of each nascent discrete vortex is determined by the vorticity flux passing through a surface element $\mathrm{d} \eta$ at the interface in a time interval. Let $\Delta \Gamma$, be the strength of the $j$ th nascent vortex in a $(i, j)$ mesh-point system. Then it may be approximately expressed as

$$
\Delta \Gamma_{j}=\int_{j} \omega U_{n} \mathrm{~d} \eta \Delta t=\frac{1}{4}\left(\omega_{j}+\omega_{j+1}\right)\left(U_{i, j}+U_{i, j+1}\right) \Delta \eta \Delta t,
$$

where $U_{n}$ is the normal velocity at the interface, $\Gamma_{j}$ has been normalized by $U_{\infty} R$.

Assume that the nascent discrete vortex issues from the center of each small arc element at the interface with an average velocity over two neighboring points $j$ and $j+1$, i. e. $U=\frac{1}{2}\left(U_{j}+\right.$ $\left.U_{j+1}\right), V=\frac{1}{2}\left(V_{j}+V_{j+1}\right)$. Then after a time step, the vortex will be located at

$$
\begin{aligned}
& \xi(t+\Delta t)=\xi\left(t, i_{1}\right)+\delta \xi, \\
& \eta(t+\Delta t)=\eta\left(t, j+\frac{1}{2}\right)+\delta \eta
\end{aligned}
$$

and

$$
\delta \xi=\frac{1}{2} U \cdot \Delta t / E, \delta \eta=\frac{1}{2} V \cdot \Delta t / E .
$$

The movement of the vortex particles already in the outer domain can be calculated by a first-order Euler scheme by VIC method. Velocity components of a vortex particle can be obtained from velocities at neighboring mesh points by using area-weighted interpolation method. When the vortex with strength $\Gamma$ reaches its new location, the corresponding vorticity distribution at mesh points is also determined by the area-weighted interpolation. Calculation of vortex convection is performed in a coarse mesh-point system ${ }^{[8]}$.

\section{Results}

Based on the method described above, three typical unsteady viscous flows around a circular cylinder at $R e=3000,5000,9500$ were calculated respectively. Transition properties were studied by flow pattern evolution and numerical analyses. To examine the accuracy and validity of the present method, the starting flows and the global features of the vortex shedding flow (such as Strouhal number, mean value of drag coefficients) for the three cases were calculated first, and compared with previous experimental data and other numerical results as well as the N-S equations' solutions for the entire flow field provided by the present work. The stability of the solutions against the shift of inner domain size was examined. The parameters used in long-time computations are as follows: at $R e=3000$ and 5000 , the size of the inner domain $r_{i} / R=4.81$; the 
fine mesh points in the domain are $128 \times 512$; the computed size of the outer domain $r_{\infty} / R$ $=23.14$; the corresponding coarse mesh points are $128 \times 256$; at $R e=9500, r_{i} / R=8.10$, $r_{\infty} / R=64.10$; the fine mesh points are $256 \times 512$ and the coarse ones are $256 \times 256$; the time step for all cases is 0.01 .

The computed results exhibit three typical flow patterns, i.e. type $\alpha$, type $\beta$ and a transition type from $\beta$ to $\alpha$, corresponding to Reynolds number 3000 , 9500 and 5000 respectively. Comparison of the flow structures at different instants given by numerical and experimental visualization of Bouard and Coutanceau ${ }^{[11]}$ are found to be excellent, especially at higher Reynolds numbers; usually it is difficult to obtain. Variations of the separation and the radial velocity with time on the symmetric axis behind the cylinder are in good agreement with previous measured data and numerical results ${ }^{[12,13]}$. In ref. [12] a fourth-order compact scheme and a second-order alternating direction implicit scheme are used to solve the Poisson equation of the stream function and the vorticity transport equation. Now the present inner solutions with fine grids and the conventional second-order A.D. I. scheme can also supply correct results. The calculated flow patterns for two different inner domain sizes and grids are found very close to each other. The solution is stable for variation of the computation parameters.

Strouhal numbers' $S_{t}$, at various Reynolds numbers are in good agreement with the experimental data given by Lienhard ${ }^{[14]}$. The computed mean values of the drag coefficient are close to the experimental results but a little bit higher, which, as pointed by Graham ${ }^{[15]}$, is mainly due to the restriction of the two-dimensional numerical approach where the three-dimensionality of the real flows is not taken into account ${ }^{[16]}$. Hence not only the starting flow but also the global features of the flows are correctly predicted by the present numerical method under the two-dimensional assumption.

2.1 Numerical visualization of nonlinear interactions of two kinds of vortex and the transition waves

Our long time computations show clearly the features of the near wall flow such as flow separation, merging, pairing and tearing of vortices at different scales. These features are difficult to be visualized in detail in experiments at present. Also the computed flow patterns and velocity fields show apparently the development of small eddy and the transition wave as well as the multiinteractions between two kinds of vortex. Flow pattern in fig. 1 shows the near wake flow structures at $R e=3000$ and $t=55.5$. At the instant the separated shear layer is oscillating upward; two small eddies emerge in the upper shear layer; the separated vortex $\mathrm{TS}_{1}$ from the upper boundary layer merges with the vortex $\mathrm{L}_{1}$ from the separation of the lower boundary layer; again a new separated vortex $\mathrm{L}_{2}$ and a secondary vortex $\mathrm{LS}_{2}$ occur near the lower side separation area: Actually all these vortex developments are accompanied by interactions, which are characterized by spectral broadening. It will be addressed in the next section of the paper.

Similar features are obtained for flow at $R e=5000$. As time goes on successive small eddies appear in the separated shear layer; small eddy moves downstream and merges with adjacent main vortex and some unstable vortices occur due to local separation.

Figure 2 shows the velocity fields of the near wake at two successive time instants $(t=58.5$ 


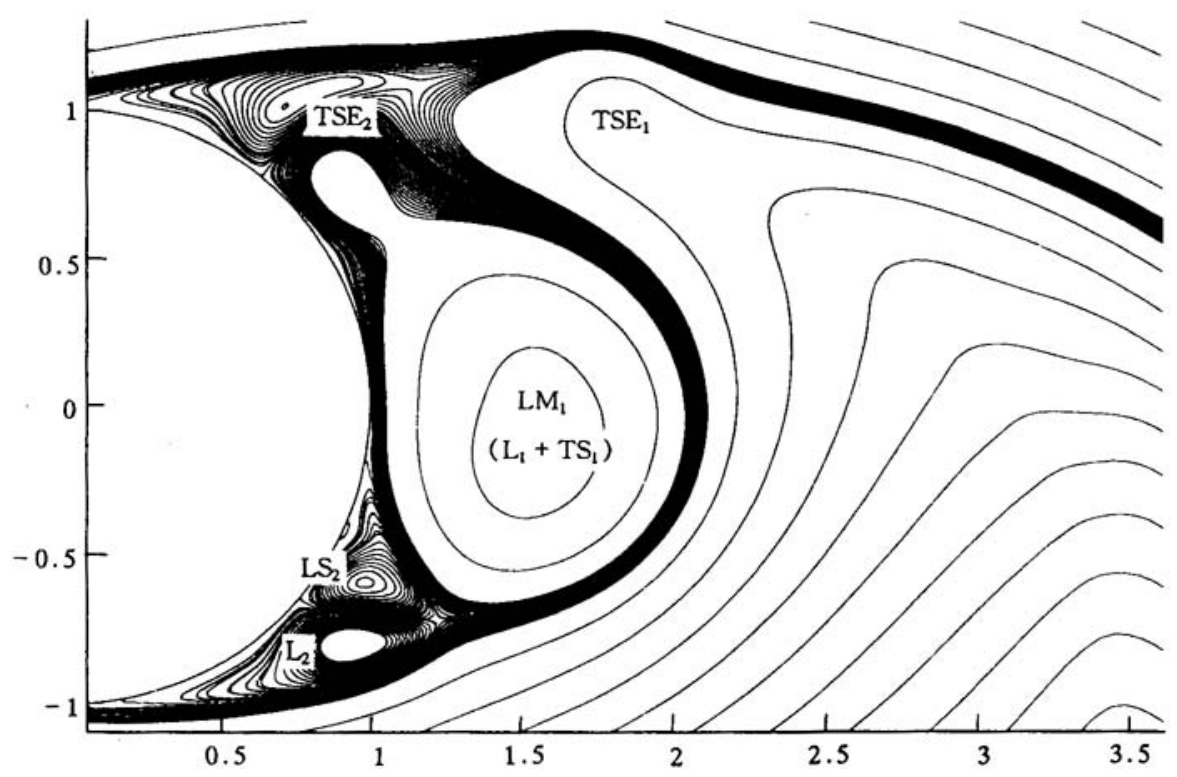

Fig. 1. Near wake flow pattern at $t=55.5, R e=3000$.

and 59) at $R e=9$ 500. It shows that a typical transition wave consists of four small eddies: $\mathrm{TSE}_{1}, \mathrm{TSE}_{2}, \mathrm{TSE}_{3}$ and $\mathrm{TSE}_{4}$. Besides, three kinds of vortex interaction are found: ( $\mathrm{i}$ ) interaction between small eddies in shear layer and the main vortex. For instance, vortex TSE ap- $_{1}$ proaches vortex TM (Fig. 2(a)) and then merges with it (Fig. 2(b)); (ii ) pairing of small eddies in the same shear layer. As shown in fig. 2(a) small eddy $\mathrm{TSE}_{3}$ follows $\mathrm{TSE}_{2}$, and at a later instant the two eddies are adjacent to each other and form a pairing vortex; (iii) interaction between vortexes in one side and opposite-side shear layers. Actually, these three kinds of interaction occur simultaneously. These multi-interactions are reflected in the velocity spectra, characterized by the generation of subharmonic frequency $1 / 2 f_{\mathrm{t}}$ and successive frequency broadening $m f_{\mathrm{s}} \pm n f_{\mathrm{t}}$.

\subsection{Numerical analyses of the transition waves and nonlinear interactions in the near wake}

For prediction of the transition features spectral analyses by fast Fourier transformation (FFT) are performed on the numerically obtained signals of the velocity components for flows around the cylinder. Since the separated mixing layer is in an oscillatory motion due to alternate vortex shedding, for accurate prediction of the transition wave and the coupling phenomena as well as their spatial variation, the numerical analyses of power spectrum with high frequency resolution for velocity are made at 12 different field points along the mixing layer in the vortex formation region. A few points on the symmetric axis of the cylinder are taken for comparison. The sampling numbers used are $N=4$ 096, dimensionless sampling time interval $\Delta T=0.05$. For comparison, $N=2048$ and $\Delta T=0.1$ are also used. The spectral resolution for these two cases is high and $\Delta f=0.0049$. The dimensionless frequencies up to 10 and 5 are provided respectively. In the examination of the influence on the evaluation of the frequency peaks from spectral resolution, the parameters are: $R e=3000, N=1024,2048, \Delta T=0.05, \Delta f=0.0195$ and 0.0098 .

At $R e=3000$, as shown in fig. 3, the spectra clearly indicate a predominant basic frequency $f_{\mathrm{s}}$ corresponding to Strouhal number $S_{\mathrm{t}}=0.22$, the transition wave frequency $f_{\mathrm{t}}$, and their har- 

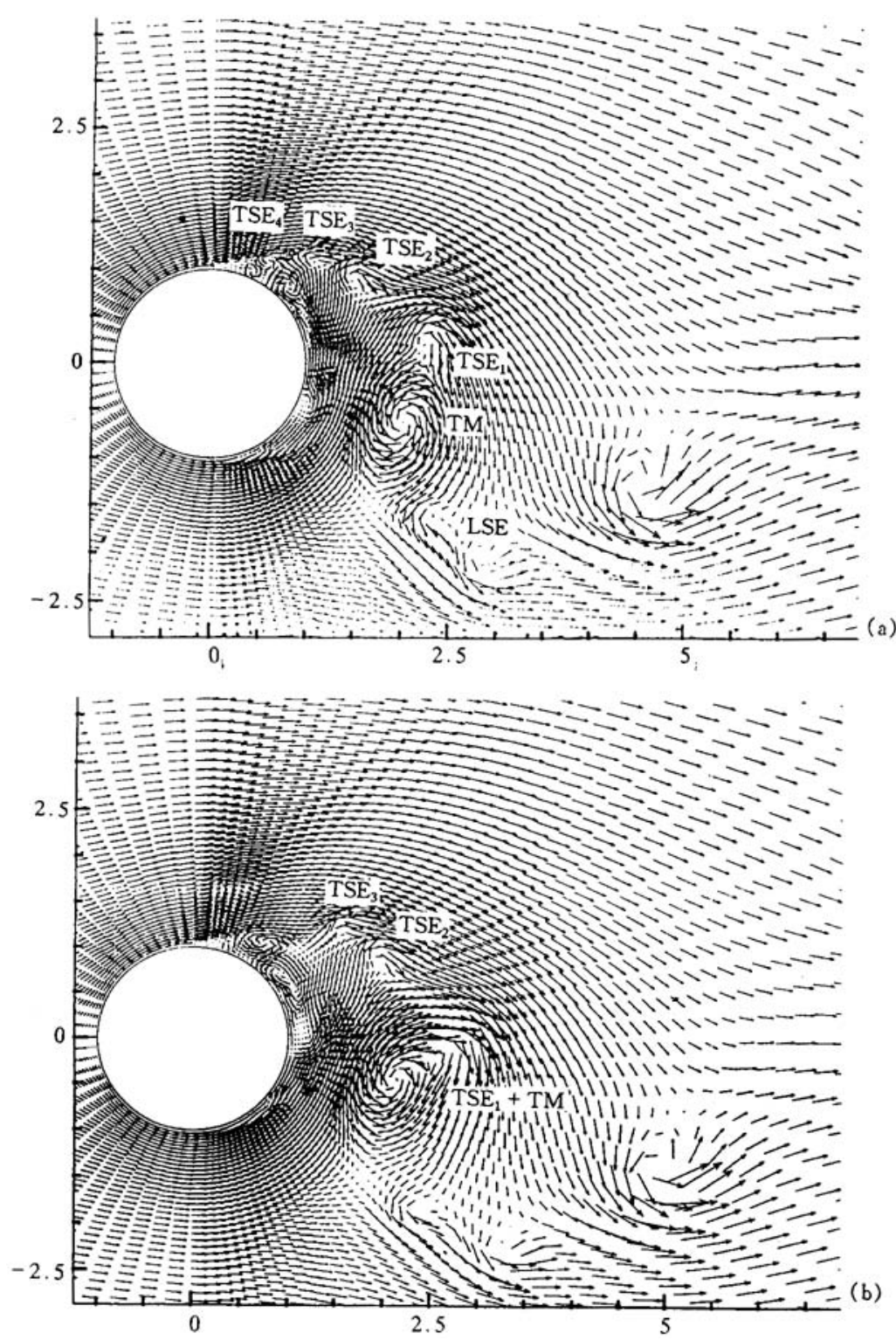

Fig. 2. The velocity fields at (a) $t=58.5$, (b) $t=59, R e=9500$.

monics $m f_{\mathrm{s}}, n f_{\mathrm{t}}$, as well as the coupling of these two independent frequencies, $m f_{\mathrm{s}} \pm n f_{\mathrm{t}}$. Except for some positions at the axis of the cylinder $(x / R=1.5, y / R=0)$ and the rear stagnation point $(1.03,0)$, where the transition wave peak is not so clearly recognized, the computed ratio $f_{\mathrm{t}} / f_{\mathrm{s}}$ from the spectra for $u$ and $v$ components are found nearly the same, being 5.03 (the error is within one $\Delta f$ ). The same results are obtained as the parameters $N=4096, \Delta T=0.05$ are used in the spectral analyses. The predicted value is in good agreement with the experimental result (5.02) of Wei and Smith provided by a relationship of $f_{\mathrm{t}} / f_{\mathrm{s}}=\left(R_{\text {ed }} / 470\right)^{0.87}$. However, it does not coincide with Braza's prediction (5.75) and experimental data (5.88) given by Bloor, who provided a 0.5 power law dependence relation.

For lower resolutions, as $\Delta f=0.0195$, only a few basic frequencies can be found. The cal- 

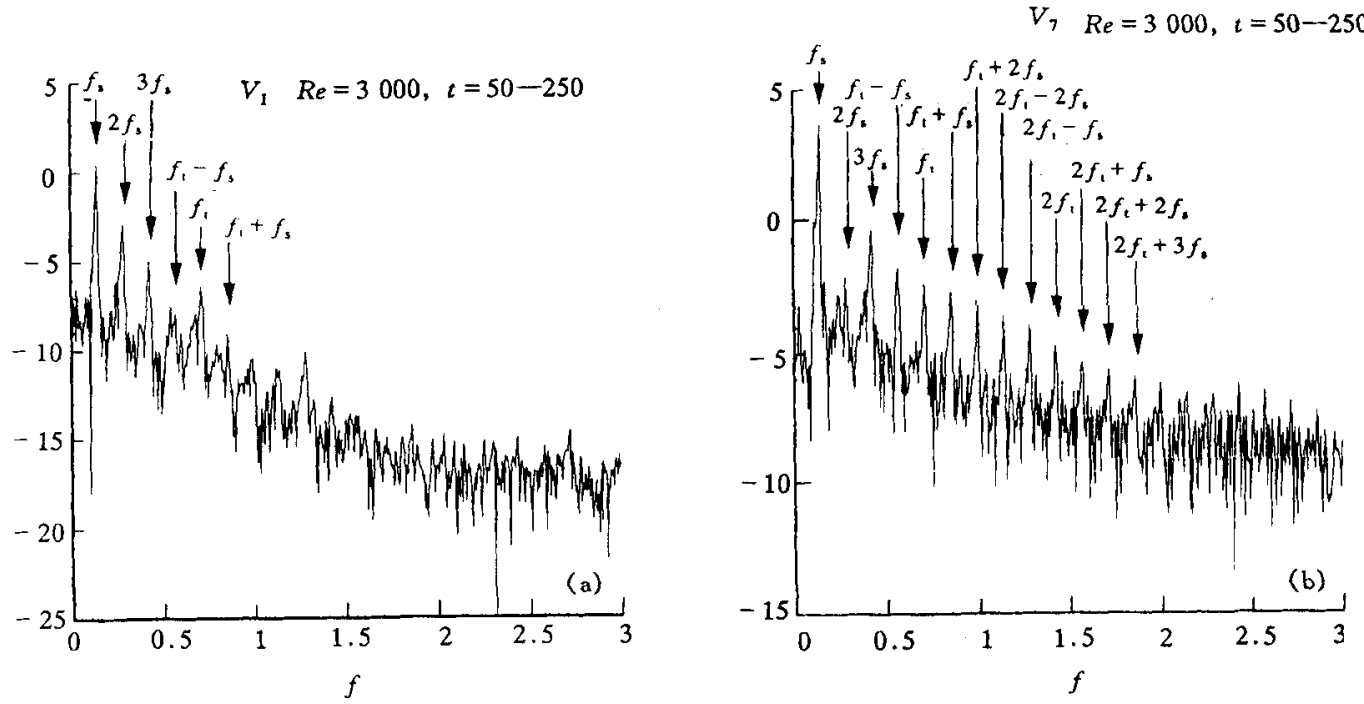

Fig. 3. The velocity $v$ component spectra. (a) At point $(x / R=0.5, y / R=1.1)$; (b) at point $(4.0,1.1), R e=3000$, $N=2048, \Delta T=0.1, \Delta f=0.0049$. The horizontal axis represents dimensionless frequency $\left(f^{\prime} R / U_{\infty}\right)$ in linear scale, the vertical axis is the spectral power in the logarithmic scale.

culated value of $f_{\mathrm{t}} / f_{\mathrm{s}}$ ranges between 4.67 and 4.83 . After the resolution increases, when $\Delta f=$ 0.0098 , frequency peaks at couplings $f_{\mathrm{s}} \pm f_{\mathrm{t}}$ appear, but higher-order couplings are still difficult to be identified. The computed value of $f_{\mathrm{t}} / f_{\mathrm{s}}$ is around 5.07. Therefore the lower resolution cannot supply an accurate evaluation of the transition features.

Figure 4 represents an example of velocity spectra at $R e=5000$. It is shown that apart from a predominant frequency $f_{\mathrm{s}}$, corresponding to $S_{\mathrm{t}}=0.205$, a clear frequency peak is $f_{\mathrm{t}}$. Some peaks at harmonic frequencies $m f_{\mathrm{s}}$ and combination $f_{\mathrm{t}} \pm m f_{\mathrm{s}}$ are also found. The value of the ratio $f_{\mathrm{t}} / f_{\mathrm{s}}$ evaluated at different points is equal to 7.24 (the error is within one $\Delta f$ ), which is in agreement with Braza's result (7.27), being close to but a little lower than the experimental results of Wei and Smith (7.82) and Bloor (7.60), higher than the value 6.72 given by Kourta $e t$ al.

At $R e=9500$ in fig. 5 the spectrum shows clearly the predominant frequency $f_{\mathrm{s}}$ (corresponding to $\left.S_{\mathrm{t}}=0.195\right)$, its harmonics, the coupling $f_{\mathrm{t}}-f_{\mathrm{s}}$ as well as the transition wave fre-

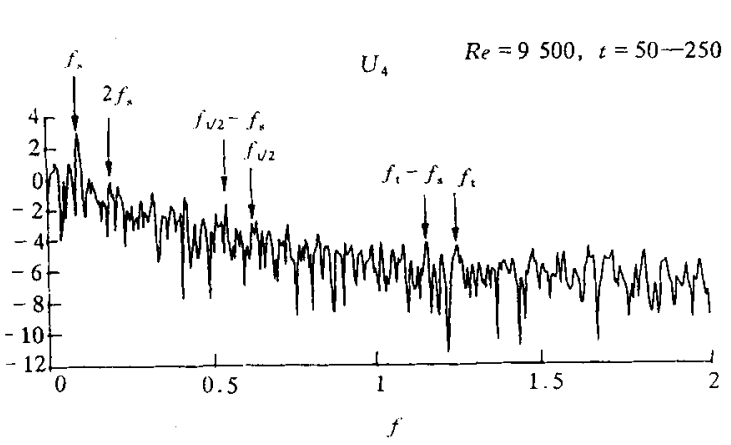

Fig. 4. The velocity $u$ componcmul spextrum at point $(x / R=$ 1.6, $y / R=1.8), R e=5000, N=4096, \Delta T=0.05$, $\Delta f=0.0049$.

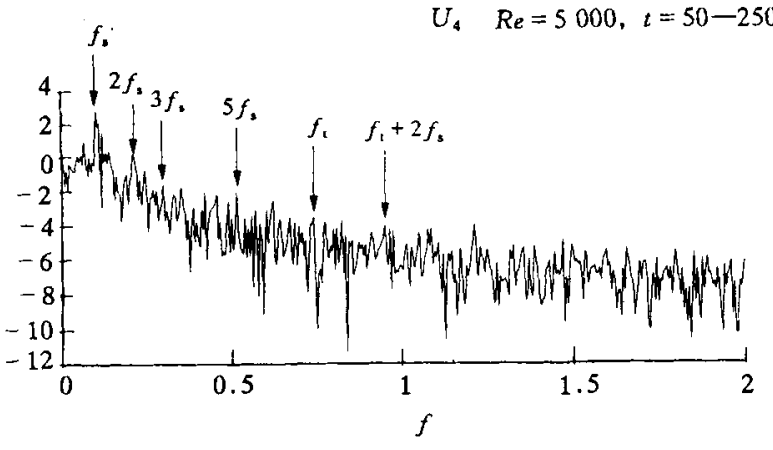

Fig. 5. The velocity $u$ component spectrum at point $(x / R=$ 2.0, $y / R=1.1), R e=9500, N=4096, \Delta T=0.05$, $\Delta f=0.0049$. 
quency $f_{\mathrm{t}}$ at a higher value. Besides, peaks at subharmonic frequency $\frac{1}{2} f_{\mathrm{t}}$ and the coupling between the subharmonic and the basic frequency are clearly shown. The appearance of the subharmonic $\frac{1}{2} f_{1}$ shows the existence of small eddy pairing in the separated mixing layer as visualized numerically in fig. 2 (b). The values of the ratio $f_{\mathrm{t}} / f_{\mathrm{s}}$ obtained from $u$ component spectra at six points are nearly the same but with a little difference. The mean value of the ratio $f_{\mathrm{v}} / f_{\mathrm{s}}$ over the six predicted results is 13.06 , and from $v$ component spectra it is 12.99 . Taking the average over all of these results, the mean value of $f_{\mathrm{t}} / f_{\mathrm{s}}$ is 13.03. The predicted value is quite close to the experimental result 13.67 of Wei and Smith, and is much greater than the numerical prediction of Braza et al. and experimental result of 9.26 suggested by Kourta et al. by 0.5 power law dependence. Comparison with the measured results is shown in figure 6 .

All comparisons in figs. 6 and 7 clearly show that

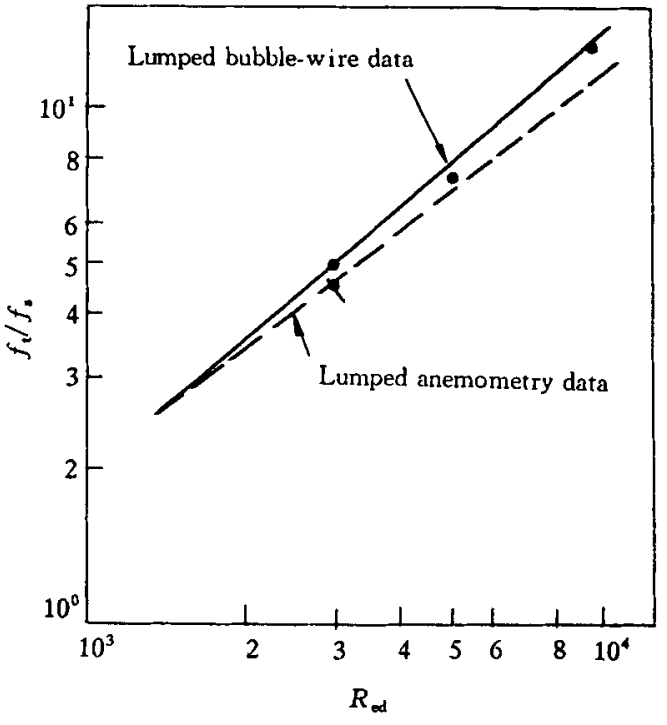

Fig. 6. Comparison of the ratio of the non-dimensional transition wave frequency over Strouhal vortex frequency versus Reynolds number (see fig. 13 of Wei \& Smith $\left.^{[s]}\right)$. $, \Delta f=0.0049, \Delta f=0.0195$ present results; - $f_{\mathrm{t}} / f_{\mathrm{s}}=\left(R_{\mathrm{ed}} / 470\right)^{0.87} ;-\cdots, f_{\mathrm{t}} / f_{\mathrm{s}}$ $=\left(R_{\mathrm{ed}} / 409\right)^{0.773[5]}$.

the present prediction of the Reynolds number dependence of the transition wave frequency agrees well with the experimental results of Wei and Smith, rather than the numerical prediction of Braza and the 0.5 power law relation suggested by Bloor and Kourta et al., i.e.

$$
f_{\mathrm{t}} / f_{\mathrm{s}}=\left(R_{\text {ed }} / 470\right)^{0.87} \text {. }
$$

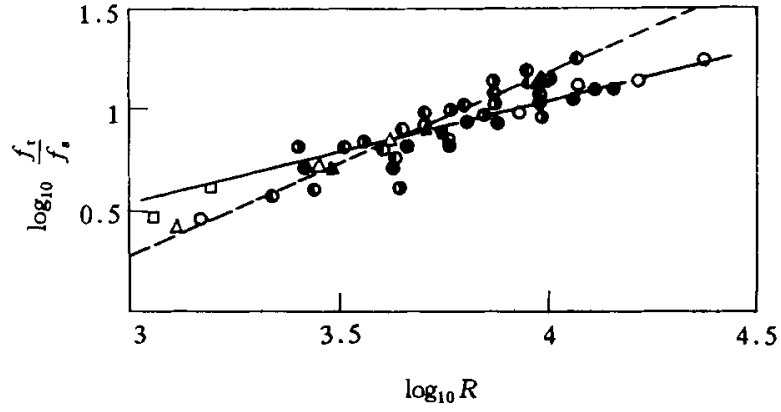

Fig. 7. The frequency ratio as a function of Reynolds number. $\Delta$, O, Bloor (1964); $\square$, Gerrard ${ }^{[17]} ;$ D. Wei \& Smith ${ }^{[5]}$;

, Kourta et al. ${ }^{[6]} ; \boldsymbol{A}$, present study. -,$f_{\mathrm{t}} / f_{\mathrm{s}} \sim R e^{0.5}$; ,$--- f_{\mathrm{t}} / f_{\mathrm{s}} \sim \operatorname{Re}^{0.87[6]}$.

tion. Moving downstream further, except the basic frequency, the strength of the transition wave and its coupling $m f_{\mathrm{s}} \pm n f_{\mathrm{t}}$ return to the low level. Fig. 8 provides a preliminary description of the longitudinal evolution of the transition wave. From the separation to a position near $x / R=1.5$ the power of the transition wave frequency $f_{\mathrm{t}}$ and the difference frequency $f_{\mathrm{t}}-f_{\mathrm{s}}$ increase exponentially. After continuous increase the power of $f_{\mathrm{t}}$ reaches the maximum near $x / R=2.6$, and 
then decreases rapidly. In view of the variation of the power intensity, the transition wave in the mixing layer and the strong interaction between the two instabilities occur pronouncedly within a downstream region of four times the radius behind the cylinder. This prediction agrees in general with the concept that the transition occurs mainly in the vortex formation region. The calculations also show that the increase of the power of the difference frequency $f_{\mathrm{t}}-f_{\mathrm{s}}$ is faster than that the transition wave frequency $f_{\mathrm{t}}$, and the power value at $f_{\mathrm{t}}-f_{\mathrm{s}}$ can be higher than that of $f_{\mathrm{t}}$ itself in the downstream. This also characterizes the nonlinear interaction in the near wake. The initial increase rate of the harmonic difference $2 f_{\mathrm{t}}-2 f_{\mathrm{s}}$ is also higher than that of $2 f_{\mathrm{t}}$. The same behavior was obtained from the longitudinal variation of power spectra for velocity component $u$. The successive multi-sideband consisting of $f_{\mathrm{t}}$ and $f_{\mathrm{s}}$ in the near wake is very similar to that in the airfoil wake generated by nonlinear interaction of two excited frequencies observed by Miksad et al. ${ }^{[18]}$, showing important characteristics of the flow transition. The above numerical predictions are comparable with the experimental measurements of Kourta et al. for $R e=$ 2660 , which demonstrated the longitudinal evolution of $f_{\mathrm{s}}$, $f_{\mathrm{t}}$ and $f_{\mathrm{v}}\left(=f_{\mathrm{t}}-f_{\mathrm{s}}\right)$, showed that after monotonical increase the components at $f_{\mathrm{t}}, f_{\nu}$ reach the maximum at $x / R$ $=2.4$ and then decrease rapidly.

At $R e=5000$, longitudinal evolution of the calculated power spectra for velocity component $v$ along $y / R=1.1$ is also shown in fig. 8. Similar to the case of $R e=3000$, the intensity of the transition wave frequency increases monotonically to a maximum value and then decreases rapidly. The maximum values evaluated from both $u$ and $v$ spectra are

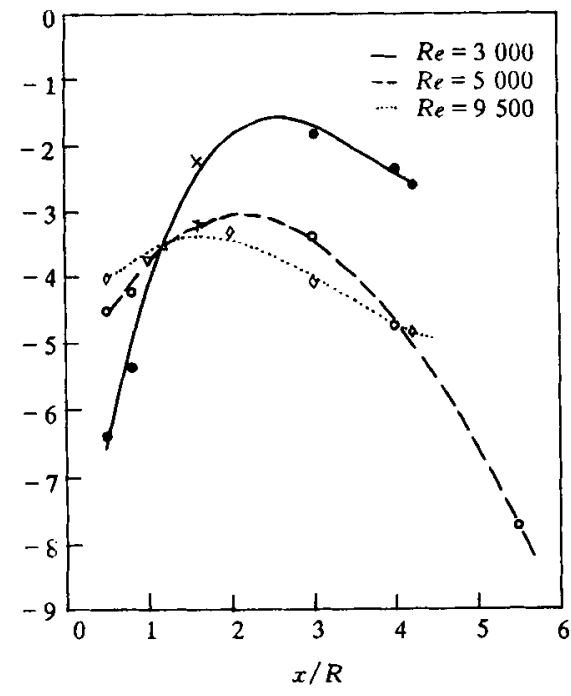

Fig. 8. Longitudinal evolution of power of the transition wave frequency (from the $v$ component), $R e=300,5000$ and 9500 , along $y / R=1.1 .(\times:(x / R, y / R)=(1.2,1$. 2), (1.6,1.8), $R e=3000 ;+,(x / R, y /$ $R)=(1.2,1.2),(1.6,1.8), R e=5000$; $\Delta,(x / R, y / R)=(1.0,1.0), R e=9500)$. nearly the same and occur at $x / R=2.2$ approximately, which move little upstream as compared with that at $R_{e}=3000$. The size of the region where the intensity of the transition wave is found stronger is also nearly four times the radius behind the cylinder. At $R e=9500$, calculation of the transition wave at various field points displays similar behavior of spatial variation as shown in fig. 8. Spectra at different positions also indicate that the peak at the subharmonic frequency $f_{t} / 2$ is not amplified considerably. It means that the vortex pairing in the separated mixing layer does not necessarily cause amplification of the subharmonic frequency, which is not the same as that in free mixing layer as pointed out by Braza et al. Influence of Reynolds number on the longitudinal variations of power spectra can be found in fig. 8 too. The maximum value for the intensity of the transition wave decreases as Reynolds number increases, and the location corresponding to the maximum tends upstream. This tendency qualitatively agrees with previous experimental results.

\section{Conclusion}

Transition features and temporal and spatial variations of the interactions between two kinds of instability - vortex shedding and the transition wave in the wake of a circular cylinder are 
studied by the domain decomposition hybrid method at $R e=3000-10000$. These complex phenomena are clearly visualized in the calculated flow patterns. The computed ratios of the dimensionless transition wave frequency $f_{\mathrm{t}} / f_{\mathrm{s}}$ are approximately proportional to the 0.87 power law of the Reynolds number. The new results agree well with the experimental results of Wei and Smith, and do not agree with numerical prediction of Braza et al. and the 0.5 power law dependence. Our calculations show that lower resolution in spectra analysis cannot supply a correct evaluation of the transition features. Nonlinear interactions between the two independent frequencies $f_{\mathrm{s}}, f_{\mathrm{t}}$ leading to spectra broadening in the near wake are described. The longitudinal evolution of the transition wave and the nonlinear interactions are reported. These new results offer detailed mechanisms for better understanding the transition behavior before occurrence of the three-dimensional evolution.

\section{Acknowledgement The first author wishes to thank the Department of Mechanical Engineering, the University of} Hong Kong for supporting the study during his academic visit in the department.

\section{References}

1 Crausse, E., Thèse dese Docteur ès sciences, Toulouse: Université de Toulouse, 1936.

2 Roshko, A., On the development of turbulent wakes from vortex streets, NACA Tech. Note, 1953, 2913.

3 Gerrard, J.H., The wake of cylindrical bluff body at low Reynolds number, Philos. Trans. .R. Soc. London, Ser. A, 1978, 288:358.

4 Bloor, M.S., The transition to turbulence in a wake of circular cylinder, J. Fluid Mech., 1964, 19:290.

5 Wei, T., Smith, C. R., Secondary vortices in the wake of circular cylinders, J. Fluid Mech., 1986, 169:513.

6 Kourta, A., Boisson, H.C., Chassaing, P. et al., Nonlinear interaction and the transition to turbulence in the wake of a circular cylinder, J. Fluid Mech., 1987 181:141.

7 Braza, M., Chassaing, P., Ming, H. Ha., Prediction of large-scale transition features in the wake of a circular cylinder, Phys. Fluid, 1990, Vol.8, No. A2.

8 Ling, G.C., Ling, G.P., Wang, Y.P., Domain decomposition hybrid method for numerical simulation of bluff body flow, Science in China, Ser. A, 1992, 35:8.

9 Ling Guo Can, Numerical study of bluff body flow structures, Key-Note Lecture, in Proceedings of 5th ACFM, Taejon, 1992; Sädhanā, Vol. 18, parts 3 \& 4 , Aug., 1993.

10 Stansby, P. K. . Dixon, A. G., Simulation of flows around cylinders by a Lagrangian vortex scheme, Applied Ocean Research, $1983,5: 167$.

11 Bouard, R., Coutanceau, M. , The early stage of development of the wake behind an impulsively started cylinder for $40<R e<$ $10^{4}, J$. Fluid Mech., 1980, $101: 583$.

12 Ta Phuoc loc, Bouard, R., Numerical solution of the early stage of the unsteady viscous flow around a circular cylinder: a comparison with experimental visualization and measurements, J. Fluid Mech., 1985, 160:93.

13 Chang, C. C., Chern, R. L., A numerical study of flow around an impulsively started circular cylinder by a deterministic vortex method, J. Fluid Mech., 1991, 233:243.

14 Lienhard, J.H., Synopsis of lift, drag, and vortex frequency data for rigid circular cylinders, Research Division Bulletin, 1996, No. 300 .

15 Schlichting, H., Boundary Layer Theory, 6th ed., New York: McGraw-Hill, 1968, 16.

16 Graham, J.M. R., Bluff-Body, W., Dynamics and Instabilities, IUTAM Symposium, Göttingen, 1992.

17 Gerrard, J.H., The mechanics of the formation region of vortices behind bluff bodies, J. Fluid Mech., 1966,25:401.

18 Miksad, M. J., Jones, F. L., Powers, E.J. et al., Experiments on the role of amplitude and phase modulations during transition to turbulence, J. Fluid Mech., 1982, $123: 1$. 